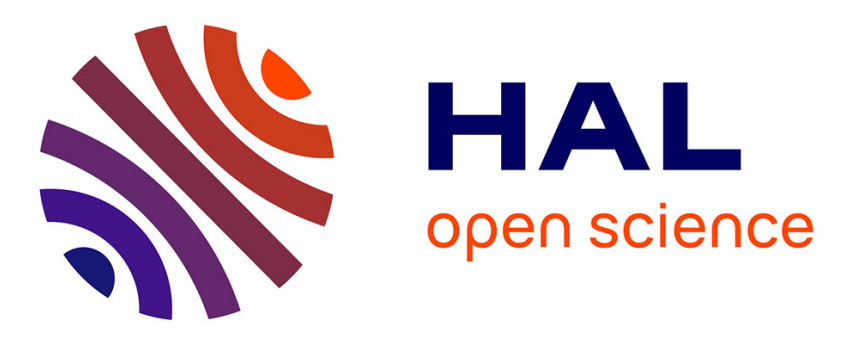

\title{
Considerations on unstirred path effects on antenna characterization in non-chaotic reverberation chambers
}

Wafa Krouka, Adnane Labdouni, Francois Sarrazin, Elodie Richalot, Philippe Besnier

\section{- To cite this version:}

Wafa Krouka, Adnane Labdouni, Francois Sarrazin, Elodie Richalot, Philippe Besnier. Considerations on unstirred path effects on antenna characterization in non-chaotic reverberation chambers. 2021 IEEE Conference on Antenna Measurements \& Applications (CAMA), Nov 2021, Antibes Juan-lesPins, France. pp.38-40, 10.1109/CAMA49227.2021.9703559 . hal-03576233

\section{HAL Id: hal-03576233 \\ https://hal.science/hal-03576233}

Submitted on 15 Feb 2022

HAL is a multi-disciplinary open access archive for the deposit and dissemination of scientific research documents, whether they are published or not. The documents may come from teaching and research institutions in France or abroad, or from public or private research centers.
L'archive ouverte pluridisciplinaire HAL, est destinée au dépôt et à la diffusion de documents scientifiques de niveau recherche, publiés ou non, émanant des établissements d'enseignement et de recherche français ou étrangers, des laboratoires publics ou privés. 


\section{Considerations on unstirred path effects on antenna characterization in non-chaotic reverberation chambers}

\author{
W. Krouka, A. Labdouni, F. Sarrazin, \\ E. Richalot \\ ESYCOM lab, UMR CNRS 9007 \\ Université Gustave Eiffel \\ F-77454 Marne-la-Vallée, France \\ elodie.richalot-taisne@univ-eiffel.fr
}

\author{
P. Besnier \\ IETR, UMR CNRS 6164 \\ INSA de Rennes \\ Rennes, France
}

\begin{abstract}
Reverberation chambers (RCs) are now widely used for the estimation of various antenna parameters such as radiation efficiency, gain, diversity gain, etc. The accuracy of such measurement relies on the assumption of a well-stirred field within the cavity. This paper evaluates the contribution of remaining unstirred path components as a function of the antenna position and the cavity wall shape. It shows their impacts on the estimated properties of an antenna, and discusses the ways to reduce their effects.
\end{abstract}

Keywords - Antenna measurement, reverberation chamber, reflection coefficient

\section{INTRODUCTION}

Beside electromagnetic compatibility testing, reverberation chambers (RCs) are now very attractive for antenna characterization in order to retrieve various parameters such as free-space impedance [1], gain radiation pattern [2] and radiation efficiency [3], [4]. Antenna characterization methods in RCs rely on the assumption of a diffuse field within the cavity. However, unstirred paths, i.e., paths that do not interact with the stirrer paddles, may exist and thus imply a deviation from the ideal measurement conditions. The estimation of several antenna parameters, such as its reflection coefficient or radiation efficiency, is impacted by this non-ideal measurement environment [5]. In this paper, we aim at showing their impacts on the estimated parameters and proposing some solutions to improve antenna characterization accuracy. First, we examine he antenna reflection coefficient, on which rely other characteristics as the antenna radiation efficiency [4]. Second, we consider the estimation of unstirred energy to stirred energy, as seen from a single antenna. Both estimations allow drawing some conclusions about the impacts of antenna position and of the shape of the wall the antenna is pointing at.

\section{REFLECTION COEFFICIENT ESTIMATION}

\section{A. Reflection coefficient model}

The reflection coefficient of an antenna measured within an $\mathrm{RC}$ results from the combined effects of the antenna mismatch and the RC backscattering towards the antenna. The $\mathrm{RC}$ response, ideally associated to a diffuse field (related to multiple reflections and diffractions within the cavity), also includes the contribution of specular reflections depending on the measurement configuration. The reflection coefficient measured for a given position $k$ of the mode stirrer can thus be written as follows [6]:

$$
S_{11, k}(f)=S_{11}^{f s}(f)+S_{11}^{\text {uns }}(f)+S_{11, k}^{s t i}(f)
$$

where $S_{11}^{f s}$ is the antenna intrinsic reflection coefficient that would be measured in free space or in an anechoic chamber (AC), $S_{11}^{\text {uns }}$ corresponds to the impact of unstirred paths whereas $S_{11, k}^{s t i}$ is associated with stirred paths and varies versus stirrer positions.

Whereas the estimation of antenna intrinsic reflection coefficient is classically performed through averaging, over a stirrer rotation, the measured reflection coefficients, the estimated parameter differs from the free-space one in the presence of unstirred paths, even for a large number of uncorrelated samples, as the ensemble average is given by :

$$
\left\langle S_{11, k}(f)\right\rangle=S_{11}^{f s}(f)+S_{11}^{\text {uns }}(f)+\left\langle S_{11, k}^{s t i}(f)\right\rangle
$$

The difference between the mean reflection coefficient measured in an $\mathrm{RC}$ and the one measured in an $\mathrm{AC}$ will be used in the following as an indicator of the unstirred component amplitude:

$$
\Delta S=\left|\left\langle S_{11, k}\right\rangle-S_{11}^{A C}\right|=\left|S_{11}^{\text {uns }}+\left\langle S_{11, k}^{s t i}\right\rangle\right|
$$

\section{B. Measurement configurations}

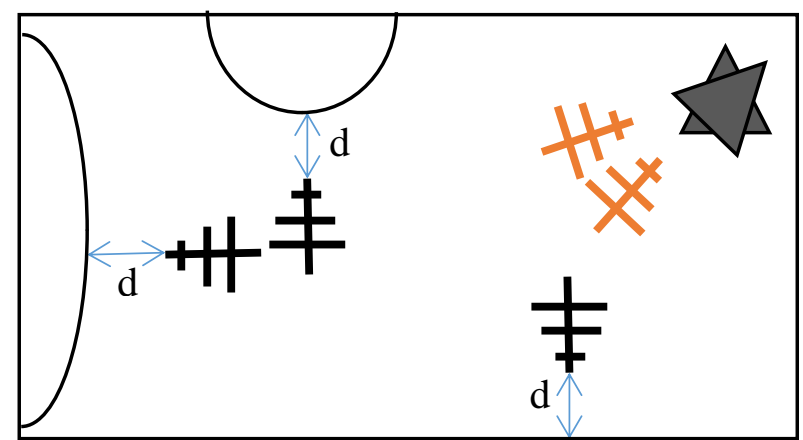

Fig. 1. Top-view of the measurement environnement with the two orientations of the excitation antenna (first antenne, in orange) and the three orientations of the measurement antenna (second antenna, in black).

To outline the presence of unstirred paths and show their effects on the estimated antenna parameters, measurements have been performed within an RC with walls of different shapes (Fig. 1). The ESYCOM RC of dimensions $2.95 \times 2.75 \times 2.35 \mathrm{~m}^{3}$ has been modified by inserting a large metallic cap (of diameter $1.8 \mathrm{~m}$ and protrusion $27 \mathrm{~cm}$ ) on one wall, and a metallic hemisphere (of radius $40 \mathrm{~cm}$ ) on an adjacent wall. Two identical directive antennas (log-periodic antennas) have been inserted within the cavity and their Sparameters have been measured using a VNA. The first antenna (in orange in Fig. 1) is pointed towards the 
mechanical mode stirrer, whereas the second one is pointed towards the RC walls of different shapes in order to bring out the presence of unstirred paths. The distance $d$ between the second antenna and the walls varies from $50 \mathrm{~cm}$ to $90 \mathrm{~cm}$ with a $10 \mathrm{~cm}$ step (Fig. 1). The reflection coefficient of the antenna is measured in the [1.2-3.2] GHz frequency range using 10,001 frequency points and 72 equally spaced stirrer positions. For each position of the second antenna, 2 orientations of the first one are considered, in order to increase the number of measurement samples. For comparison purposes, the reflection coefficient of the second antenna is measured in the same frequency range within an AC.

\section{Measurement results}

The ability of $\Delta \mathrm{S}$ indicator to reflect the contribution level of the unstirred component is firstly shown by its decrease with the distance between the antenna and the facing wall, for a chosen wall shape. Fig. 2 presents the values of this indicator versus frequency for three different distances from the flat wall, indicating a clear decrease when the distance increases. The same trend is observed when the antenna points towards the metallic cap (Fig. 3) and the metallic hemisphere (Fig. 4). We can notice the decrease of $\Delta \mathrm{S}$ versus frequency due to the increase of the direct antenna-wall path length in terms of wavelengths. Besides, a decrease of this error versus the distance from the wall can be noticed for the three orientations of the second antenna.

The mean value of $\Delta \mathrm{S}$ over the whole frequency range is then calculated at each distance from the 3 shapes of facing wall (Fig. 5). It clearly shows the reduction of the unstirred path impact while replacing the flat surface by a curved one, and also while decreasing the surface radius of curvature (from the cap to the hemisphere).

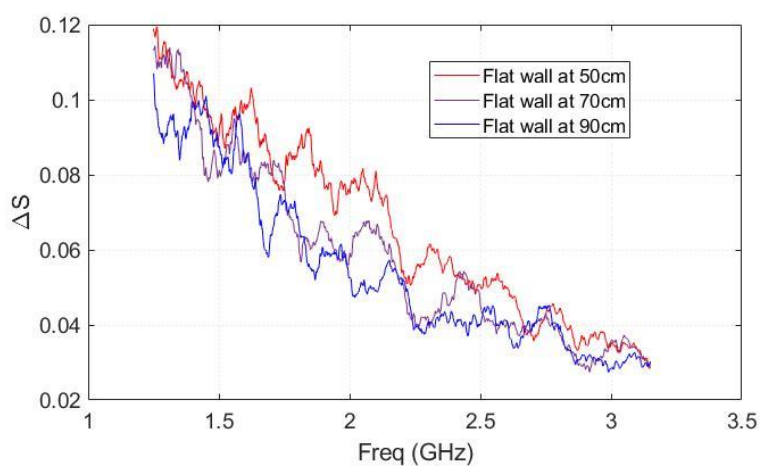

Fig. 2. $\Delta \mathrm{S}$ for the antenna pointing towards the flat wall, at three distances from the wall. Results smoothed over a frequency window of $100 \mathrm{MHz}$.

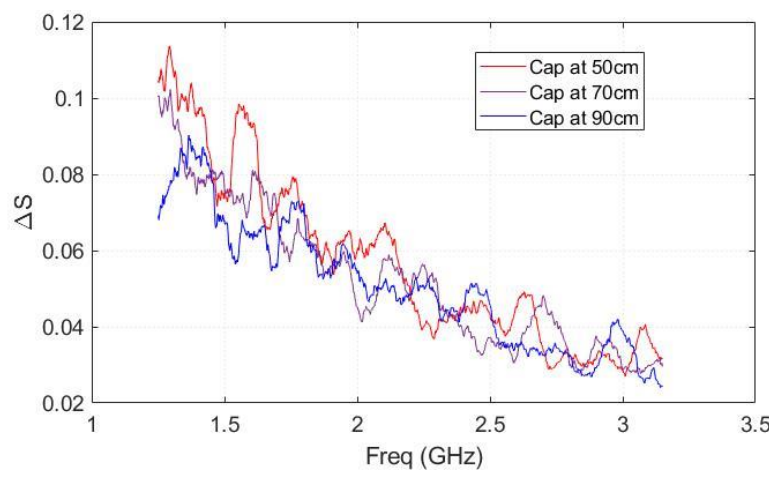

Fig. 3. $\Delta \mathrm{S}$ for the antenna pointing towards the metallic cap, at three distances from it. Results smoothed over a frequency window of $100 \mathrm{MHz}$.

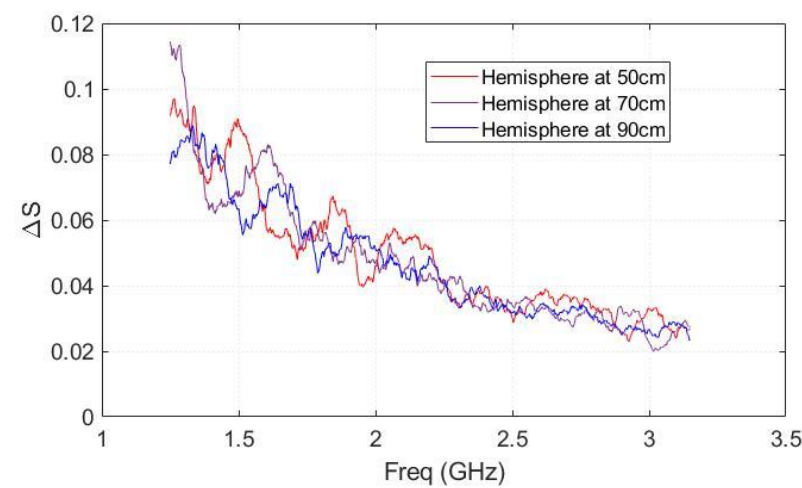

Fig. 4. $\Delta \mathrm{S}$ for the antenna pointing towards the metallic hemisphere, at three distances. Results smoothed over a frequency window of $100 \mathrm{MHz}$.

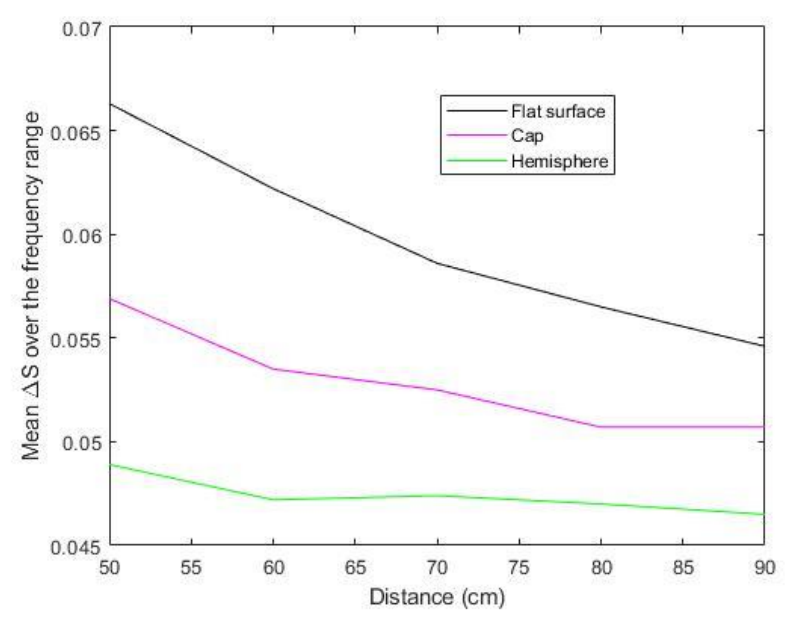

Fig. 5. Mean value of $\Delta \mathrm{S}$ over the [1.2-3.2] GHz frequency range for the antenna orientations and the 5 antenna-wall distances.

Another way to estimate the contribution of the unstirred path can consist in the comparison of the energy related to the unstirred path compared to the one associated to the diffuse field. This approach is inspired from the one adopted to evaluate the direct path amplitude between two antennas within an RC through the estimation of the Rician K-factor [7]. It has to be noticed that the amplitude of the unstirred energy is about twice of the one obtained in the case of a transmission between both antennas.

In the present paper, both energies are compared according to:

$$
K=\frac{\left|S_{11}^{u n s}\right|^{2}}{\left\langle\left|S_{11, k}^{s t i}\right|^{2}\right\rangle}
$$

where both terms are estimated by:

$$
\begin{gathered}
S_{11}^{u n s}=\left\langle S_{11, k}-S_{11}^{A C}\right\rangle \\
S_{11, k}^{s t i}=S_{11, k}-S_{11}^{f s}-\left\langle S_{11, k}-S_{11}^{A C}\right\rangle
\end{gathered}
$$

It has to be noticed that the energy related to the stirred reflection coefficient, that is in particular useful to estimate the antenna radiation efficiency, is classically calculated as $S_{11, \mathrm{~s}}=S_{11}-\left\langle S_{11}\right\rangle$, and is thus not valid any more in the presence of unstirred paths within the cavity.

The $\mathrm{K}$ parameters obtained for an antenna pointing towards a flat wall or the hemisphere are shown in Figs. 6-7. They confirm a decrease of the unstirred component impact when the frequency increases and when the distance from the 
wall increases. A comparison between the values obtained at the same distance for the 3 wall geometries (Fig. 8) shows the advantage of curved surfaces to decrease this unstirred component impact. This trend is confirmed by the mean values of $\mathrm{K}$ calculated over the whole frequency range (Fig. 9): a clear decrease of $\mathrm{K}$ values is observed when the radius of curvature of the reflecting surface decreases.

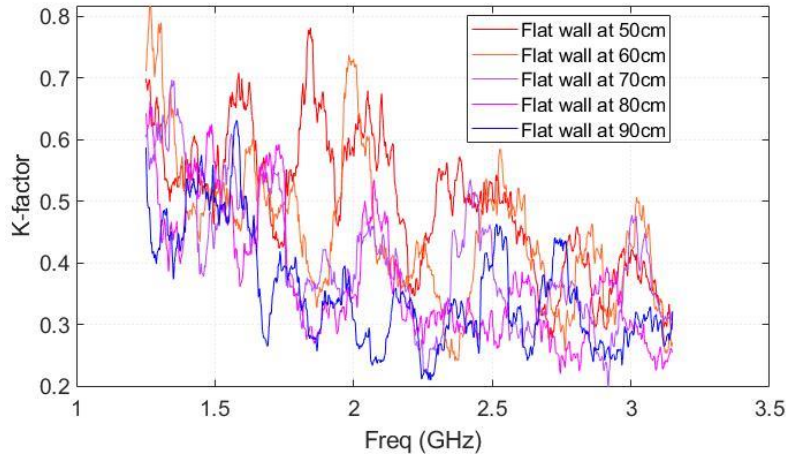

Fig. 6. Amplitudes of $\mathrm{K}$ parameter versus frequency for the antenna pointed towards the flat wall.

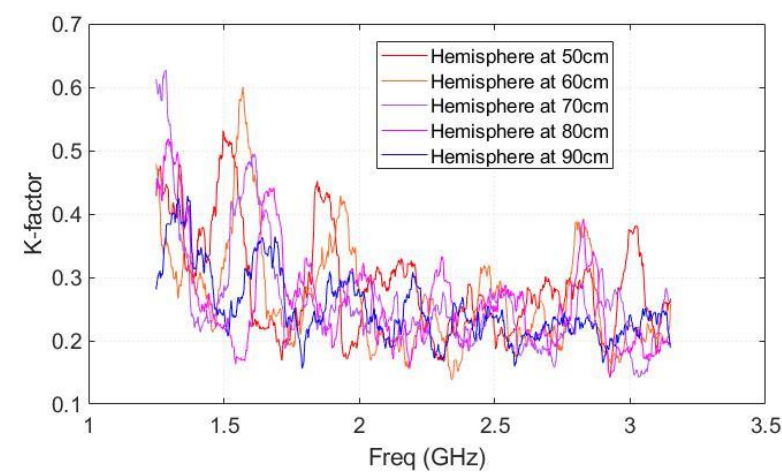

Fig. 7. Amplitudes of $\mathrm{K}$ parameter versus frequency for the antenna pointed towards the hemisphere.

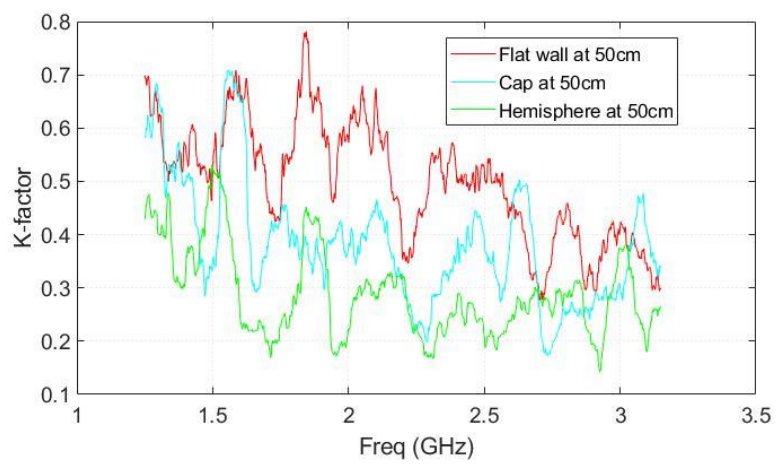

Fig. 8. Amplitudes of $\mathrm{K}$ parameter versus frequency for the antenna at $50 \mathrm{~cm}$ from the 3 types of walls.

These results confirm the conclusion drawn while observing $\Delta \mathrm{S}$ concerning the decrease of the unstirred component contribution when the distance from the wall increases and also when the radius of curvature of the reflecting surface decreases.

\section{CONCLUSION}

This paper highlights the impact of unstirred paths on the antenna reflection coefficient measured within an RC. It shows that, depending on the cavity wall geometry and the distance between the antenna and the wall, their impact is far from being negligible and their consideration is thus necessary. Several measurement configurations have been examined to highlight the most critical cases and estimate the potential discrepancy from ideal measurement conditions. Two indicators have been proposed to evaluate this effect. The first one is based on the difference between the freespace reflection coefficient estimated in the RC compared to the one measured in an AC. The second one consists in the ratio between the energies related to the unstirred paths and to the stirred ones. Both indicators agree to show the increasing impact of the unstirred paths when the antenna points towards a flat wall and when the distance to the wall decreases. These results show that avoiding flat metallic surfaces within RCs would be of interest to avoid such unstirred paths and improve the estimation reliability of antenna properties with RCs. Future works include the impact of these unstirred paths on the estimated antenna radiation efficiency.

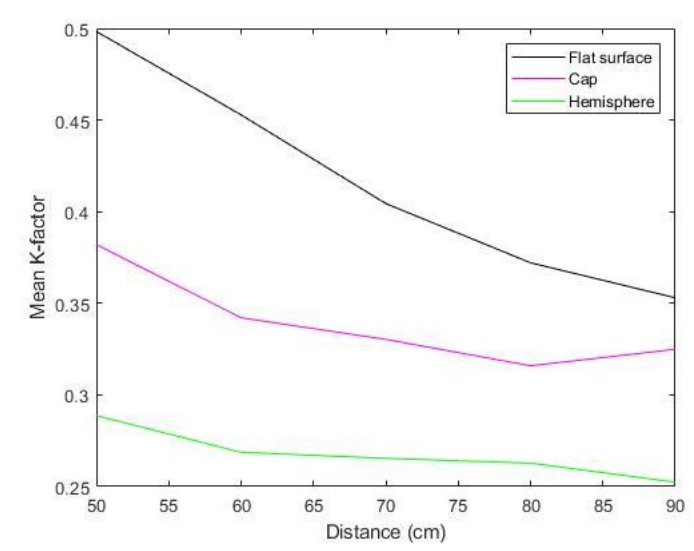

Fig. 9. Mean values of $\mathrm{K}$ parameter over the [1.2-3.2] $\mathrm{GHz}$ frequency range for the antenna orientations and the 5 antenna-wall distances.

\section{REFERENCES}

[1] P. -S. Kildal et al. "Measurement of free-space impedances of small antennas in reverberation chambers." Microwave and Optical Technology Letters 32 (2002): 112-115.

[2] A. Reis, F. Sarrazin, P. Besnier, P. Pouliguen and E. Richalot, "Contactless antenna gain pattern estimation from backscattering coefficient measurement performed within reverberation chambers," IEEE Trans. Antennas Propag., doi: 10.1109/TAP.2021.3111184.

[3] C. L. Holloway, H. A. Shah, R. J. Pirkl, W. F. Young, D. A. Hill, and J. Ladbury, "Reverberation chamber techniques for determining the radiation and total efficiency of antennas," IEEE Trans. Antennas Propag., vol. 60, no. 4, pp. 1758-1770, Apr. 2012.

[4] W. Krouka, F. Sarrazin, J. Sol, P. Besnier and E. Richalot, "Comparison of Antenna Radiation Efficiency Measurement Techniques in Reverberation Chamber Using or Not a Reference Antenna," Europ. Conf. Antennas Propag. (EuCAP), 2020, pp. 1-4, doi: 10.23919/EuCAP48036.2020.9135507.

[5] W. Krouka, F. Sarrazin and E. Richalot, "Influence of the Reverberation Chamber on Antenna Characterization Performances," Int. Symp. Electromagn. Compat. (EMC EUROPE), 2018, pp. 329333, doi: 10.1109/EMCEurope.2018.8485064.

[6] G. Andrieu, N. Ticaud, F. Lescoat, L. Trougnou, "Fast and accurate assessment of the "well stirred condition" of a reverberation chamber," IEEE Trans. Electromagne. Compat., vol. 61, pp. 974-982, Aug. 2019.

[7] C. Lemoine, E. Amador, J. Sol, P. Besnier, "K-factor as an accurate estimator of the stirring efficiency in reverberation chamber," $9^{\text {th }}$ international on EMC, Sep 2010, Wroclaw, Poland. pp.179-184. 